\title{
A wall-aligned grid generator for non-linear simulations of MHD instabilities in tokamak plasmas
}

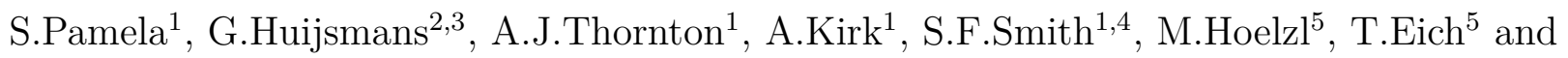 \\ JET Contributors ${ }^{6}$, the MAST Team ${ }^{1}$ and the JOREK Team ${ }^{7}$. \\ *EUROfusion Consortium, JET, Culham Science Centre, Abingdon, OX14 3DB, UK. \\ ${ }^{1}$ CCFE, Culham Science Centre, Abingdon, Oxon, OX14 3DB, UK. \\ ${ }^{2}$ CEA, IRFM, F-13108 Saint-Paul-lez-Durance, France. \\ ${ }^{3}$ Eindhoven University of Technology, Eindhoven, The Netherlands. \\ ${ }^{4}$ York Plasma Institute, Department of Physics, University of York, Heslington, York YO10 5DD. \\ ${ }^{5}$ Max-Planck-Institut für Plasmaphysik, Garching, Germany \\ ${ }^{6}$ See author list of [X.Litaudon, Nucl. Fusion 57, 102001 (2017)]. \\ ${ }^{7}$ See https://www.jorek.eu team members.
}

\begin{abstract}
Block-structured mesh generation techniques have been well addressed in the CFD community for automobile and aerospace studies, and their applicability to magnetic fusion is highly relevant, due to the complexity of the plasma-facing wall structures inside a tokamak device. Typically applied to non-linear simulations of MHD instabilities relevant to magnetically confined fusion, the JOREK code was originally developed with a 2D grid composed of isoparametric bi-cubic Bézier finite elements, that are aligned to the magnetic equilibrium of tokamak plasmas (the third dimension being represented by Fourier harmonics). To improve the applicability of these simulations, the grid-generator has been generalised to provide a robust extension method, using a block-structured mesh approach, which allows the simulations of arbitrary domains of tokamak vacuum vessels. Such boundary-aligned grids require the adaptation of boundary conditions along the edge of the new domain. Demonstrative non-linear simulations of plasma edge instabilities are presented to validate the robustness of the new grid, and future potential physics applications for tokamak plasmas are discussed. The methods presented here may be of interest to the wider community, beyond tokamak physics, wherever imposing arbitrary boundaries to quadrilateral finite elements is required.
\end{abstract}

\section{Introduction and Motivation}

As fusion devices progress towards reactor-relevant conditions, the exhaust of particles and heat onto plasma facing components (PFCs) is acknowledged to be a major challenge [1]. In JET, the tungsten divertor is subject to damage in high power discharges, and there is growing concern that 
divertor heat loads will be one of the main restricting factors for operation capabilities in ITER [2]. Energy deposition on the divertor materials, due to steady-state heat fluxes as well as transient MHD events like Edge-Localised-Modes (ELMs), will be considerably increased in burning plasma experiments like ITER. Effectively most of the energy that crosses the separatrix into the ScrapeOff Layer (SOL) is transported along field lines to the divertor targets, and this effect is enhanced by increased heat conductivity at higher temperatures [3-5]. Combined with the large amplitude of these heat-fluxes, the longer duration of experimental pulses in reactor-sized devices will induce significant strain on the tungsten tiles, possibly leading to erosion and melting [6]. The tungsten tiles of the ITER divertor are designed to withstand up to $10 \mathrm{MW} \cdot \mathrm{m}^{-2}$ in steady-state, and several thousands of transient events of up to $20 \mathrm{MW} . \mathrm{m}^{-2}[7,8]$.

Ultimately, heat fluxes on PFCs are determined by how much energy escapes from the wellconfined region inside the separatrix. It is well established that anomalous transport is responsible for this energy crossing the separatrix [9], and it is also well established that this occurs either in the form of turbulence filaments (blobs), or in the form of large-scale ELM filaments [10-13]. Hence, on one side, elaborate physics models (Fluid, MHD, drift-kinetic etc.) are indispensable to yield a robust understanding of anomalous transport in tokamaks. On the other side, the elaborate geometry of magnetic equilibria, including X-point and separatrix, is a fundamental ingredient for the accurate description of filamentary transport at the plasma edge and in the SOL. This has led a number of state-of-the-art numerical codes [14-18] to use various field- or flux-aligned grid techniques, since it is essential to resolve the large parallel energy transport when these filaments cross the separatrix. However, the extension of such grids to the entire vacuum domain of a tokamak is not trivial, and often not undertaken.

In order to provide accurate estimates of divertor and first-wall heat-fluxes in present and future tokamak simulations, it is essential to consider the exact plasma domain inside the vacuum vessel wall. The precise location of individual wall components is necessary to obtain their respective heat-fluxes, and the accuracy of the global wall geometry is also required to represent the transport of neutral particles in various regions of the plasma domain. In addition, boundary conditions on wall components that are not aligned to magnetic field lines constitute a key aspect of simulations as a whole, particularly since boundary conditions have a direct impact on the plasma dynamics in the entire SOL.

In this paper, we present a newly developed grid generator developed for the JOREK code $[19,20]$. This block-structured mesh generator enables the extension of single and double Xpoint grids to the entire vacuum domain for arbitrary tokamak configurations, and non-linear simulations of ELMs are performed to demonstrate the robustness of such wall-extended grids for the JET-ILW tokamaks. Block-structured mesh generation is a technique that has already been thoroughly addressed by the CFD community for a wide array of applications, including automobile and aerospace [21-23]. The applicability of structured meshes to magnetic fusion is particularly relevant due to the complexity of the PFCs inside tokamaks. This work is part of a wider effort in the fusion community to improve and generalise grid-generation techniques, and it is complementary 
of recent works for triangular meshes [24], and for advanced magnetic configurations [25]. The particularity of the method developed here is that it adapted to the quadrilateral topology imposed by the rectangular Bezier elements used in JOREK, and thus it may be of interest to the wider community, beyond tokamak physics.

The paper is organised as follows. Section-2 presents the JOREK code, including its bicubic Bezier finite-element formulation, and the visco-resistive MHD models used for simulations. Section-3 describes the methods used to extend flux-aligned grids to the first-vessel wall for arbitrary tokamak geometries. Section-4 discusses the use of Bohm Sheath boundary conditions [9] with wall-aligned grids. Section-5 shows initial results of ELM simulations in the JET-ILW tokamak, and discusses prospects for future studies with the new wall-extension grid generator in JOREK. This article is closed with a brief conclusion in Section-6.

\section{The JOREK Code}

\subsection{Visco-resistive MHD with neutrals density}

The 3D nonlinear MHD code JOREK was developed by Huysmans et al. with the specific aim to produce simulations of Edge-Localised-Modes [19, 26]. The MHD model used for the present paper is similar to that used in previous ELM and disruption studies [27, 28]. It is a six-field reduced MHD model for the variables $\psi$ (poloidal magnetic flux), $\Phi$ (electric potential), $\overrightarrow{\mathrm{v}}_{\|}$(parallel velocity), $\rho$ (ion density), $T$ (total temperature assuming $T_{i}=T_{e}$ ), $\rho_{n}$ (neutral density), including the two-fluid diamagnetic effects [29]. The reduction of the equations assumes that the perpendicular velocity lies in the poloidal plane, and that the toroidal magnetic field is constant in time, so that the total plasma velocity and the total magnetic field are expressed respectively as

$$
\begin{aligned}
\overrightarrow{\mathrm{v}}_{t o t} & =\overrightarrow{\mathrm{v}}_{\|}+\overrightarrow{\mathrm{v}}_{\perp}=\overrightarrow{\mathrm{v}}_{\|}+\overrightarrow{\mathrm{v}}_{E}+\overrightarrow{\mathrm{v}}_{* i} \\
& =v_{\|} \vec{B}+R \vec{e}_{\phi} \times \nabla \Phi+\frac{\delta^{*} R}{\rho} \vec{e}_{\phi} \times \nabla p_{i}, \\
\vec{B} & =\vec{B}_{\phi}+\vec{B}_{p}=\frac{F_{o}}{R} \vec{e}_{\phi}+\frac{1}{R} \nabla \psi \times \vec{e}_{\phi},
\end{aligned}
$$

where $R$ is the major radius, $\vec{e}_{\phi}$ is the toroidal unit vector and $F_{o}=B_{o} R_{o}$, with $B_{o}$ being the magnetic field amplitude at the reference major radius $R=R_{o}$. The diamagnetic component of the perpendicular velocity is represented by the third term $\overrightarrow{\mathrm{v}}_{* i}=\delta^{*} R \rho^{-1} \vec{e}_{\phi} \times \nabla p_{i}$, where $p_{i}$ is the ion pressure and $\delta^{*}=\left(\Omega_{c i} R_{o}\right)^{-1}$, with the ion gyrofrequency $\Omega_{c i}=e B_{o} / m_{i}$. Substituting the identities (1) and (2) into the visco-resistive MHD equations gives the reduced MHD model, first derived by H.R. Strauss [30], with two separate equations for the parallel and the perpendicular momentum. The complete set of normalised equations is as follows, where diamagnetic terms are highlighted in green, neutral density terms in red, and hyper-diffusive terms in blue (also with subscript $\left.\right|_{\text {hyp }}$ ): 


$$
\begin{aligned}
& \rho \frac{d \overrightarrow{\mathrm{v}}_{E}}{d t}=\quad-\rho \overrightarrow{\mathrm{v}}_{* i} \cdot \nabla \overrightarrow{\mathrm{v}}_{E}-\nabla_{\perp} p+\vec{J} \times \vec{B}+\mu \nabla^{2}\left(\overrightarrow{\mathrm{v}}_{E}+\overrightarrow{\mathrm{v}}_{* i}\right) \\
& +\left(\rho^{2} \alpha_{\text {rec }}-\rho \rho_{n} S_{\text {ion }}\right) \overrightarrow{\mathrm{v}}_{E}+\mu_{\text {hyp }} \nabla^{4} \overrightarrow{\mathrm{v}}_{E} \text {, } \\
& \rho \frac{d \overrightarrow{\mathrm{v}}_{\|}}{d t}=\quad-\rho \overrightarrow{\mathrm{v}}_{\|} \cdot \nabla \overrightarrow{\mathrm{v}}_{\|}-\nabla_{\|} p+\mu \nabla^{2}\left(\overrightarrow{\mathrm{v}}_{\|}-V_{N B I}\right) \\
& +\left(\rho^{2} \alpha_{r e c}-\rho \rho_{n} S_{i o n}\right) \overrightarrow{\mathrm{v}}_{\|}+\mu_{\text {hyp }} \nabla^{4} \overrightarrow{\mathrm{v}}_{\|}, \\
& \frac{\partial \psi}{\partial t}=\quad \eta\left(j-j_{A}\right)+R[\psi, \Phi]-\frac{\partial \Phi}{\partial \phi} \\
& -\frac{\delta^{*} R}{\rho}\left[\psi, p_{e}\right]+\frac{\delta^{*}}{\rho} \frac{\partial p_{e}}{\partial \phi}+\eta_{h y p} \nabla^{2} j \\
& \frac{\partial \rho}{\partial t}=\quad-\nabla \cdot\left(\rho\left[\overrightarrow{\mathrm{v}}_{\|}+\overrightarrow{\mathrm{v}}_{E}+\overrightarrow{\mathrm{V}}_{* i}\right]\right)+\nabla \cdot\left(D_{\perp} \nabla_{\perp} \rho\right)+S_{\rho} \\
& +\left(\rho \rho_{n} S_{i o n}-\rho^{2} \alpha_{r e c}\right)+D_{\text {hyp }} \nabla^{4} \rho \text {, } \\
& \frac{\partial p}{\partial t}=\quad-\overrightarrow{\mathrm{v}}_{E} \cdot \nabla p-\gamma p \nabla \cdot \overrightarrow{\mathrm{v}}_{E} \\
& +\nabla \cdot\left(\kappa_{\perp} \nabla_{\perp} T+\kappa_{\|} \nabla_{\|} T\right)+\frac{2}{3 R^{2}} \eta j^{2}+S_{T} \\
& +\xi_{\text {ion }} \rho \rho_{n} S_{\text {ion }}-\rho \rho_{n} L_{\text {lines }}-\rho^{2} L_{\text {brem }} \text {, } \\
& \frac{\partial \rho_{n}}{\partial t}=\nabla \cdot\left(\vec{D}_{n}: \nabla \rho_{n}\right)+S_{\rho}-\left(\rho \rho_{n} S_{i o n}-\rho^{2} \alpha_{r e c}\right),
\end{aligned}
$$

where the density, temperature and current sources $S_{\rho}, S_{T}$ and $j_{A}$ have been introduced. The current source term $j_{A}$ also includes the time-dependent bootstrap current calculated using Sauter's formula [31]. The convective derivative, the parallel gradient, the perpendicular gradient, and the Poisson brackets are defined as

$$
\begin{aligned}
& \frac{d}{d t}=\frac{\partial}{\partial t}+\overrightarrow{\mathrm{v}}_{E} \cdot \nabla, \\
& \nabla_{\|}=\vec{b}[\vec{b} \cdot \nabla], \\
& \nabla_{\perp}=\nabla-\nabla_{\|}, \\
& {[\alpha, \beta]=\vec{e}_{\phi} \cdot(\nabla \alpha \times \nabla \beta),} \\
& \vec{b}=\frac{1}{|B|} \vec{B} .
\end{aligned}
$$

As defined in [28], $S_{i o n}$ and $\alpha_{r e c}$ are the ionization and recombination rate coefficients for deuterium, $\xi_{\text {ion }}$ is the normalized ionization energy of a D atom (here set to $13.6 \mathrm{eV}$ ), and $L_{\text {lines }}$ and $L_{b r e m}$ are the line and bremsstrahlung radiation rate coefficients (based on ADAS data [32]). 
Note that this neutrals model is an extremely simplified description of a very complex system, without evolution equations for momentum or energy, which have been shown to play a major role in SOL physics, particularly detachment $[15,33]$. Here, the neutrals fluid spreads in a diffusive manner, which is only valid for short mean-free-path regimes. In this paper, the diffusive parameter is set to $D_{n}=10^{-4} m^{2} s^{-1}$, similar to previous works from [28, 34].

Note that equations (3) and (4) can be reduced to scalar equations by projecting them in the poloidal and parallel directions, respectively, by applying the operators $\nabla \cdot\left[R \vec{e}_{\phi} \times()\right]$ and $\vec{b} \cdot()$. This reduced set of equations (without the diffusive transport terms and the diamagnetic terms) is equivalent to that derived by HR.Strauss, where energy of the system is shown to be conserved at first order $[35,36]$.

The perpendicular mass and thermal diffusivities $D_{\perp}$ and $\kappa_{\perp}$ used in simulations are ad-hoc coefficients with a well at the pedestal region to represent the H-mode transport barrier. Spitzer resistivity $\eta=\eta_{o}\left(T_{e} / T_{e, o}\right)^{-3 / 2}$ is used, with $T_{e, o}$ the electron temperature at the magnetic axis. Likewise, a temperature-dependent perpendicular viscosity is used: $\mu=\mu_{o}\left(T_{e} / T_{e, o}\right)^{-3 / 2}$. The Braginskii parallel thermal conductivity $\kappa_{\|}$is expressed as $\kappa_{\|}=\kappa_{\| o}\left(T / T_{o}\right)^{5 / 2}$. The ratio of specific heat is $\gamma=5 / 3$. Hyper-diffusive coefficients $\mu_{h y p}, \eta_{h y p}$ and $D_{h y p}$ are also used in these simulations to improve numerical stability, but with values small enough not to influence the physics results. Typically, $\eta_{h y p} \sim \eta^{2}$ is chosen (and similarly for $\mu_{h y p}$ and $D_{h y p}$ ).

The normalization of the equations is based on the magnetic permeability $\mu_{o}$ and the core density $\rho_{o}$, so that time is normalized to a near Alfven time $t=t_{S I} / \sqrt{\mu_{O} \rho_{o}}$. For a deuterium plasma with particle density $n_{o}=6 \times 10^{19} \mathrm{~m}^{-3}$, a normalized time unit corresponds to approximately $0.5 \mu \mathrm{s}$. Naturally, the current density is normalized as $\tilde{j}=\mu_{o} j$, the density as $\tilde{\rho}=\rho / \rho_{o}$, and pressure as $\tilde{p}=\mu_{o} p$.

\subsection{Spatial and Temporal Discretization}

The spatial discretization of the JOREK code is made of a 2D grid in the poloidal plane (as shown in Figure-1), and a pseudo-spectral Fourier representation in the toroidal direction. The 2D poloidal grid is composed with isoparametric bi-cubic Bezier finite elements, as described in [26]. This finite element grid is aligned to equilibrium flux surfaces for the three regions of the core, the SOL and the private region. This alignment along flux surfaces is particularly important in the region of the separatrix, in order to treat accurately the fast parallel transport of energy along magnetic field lines.

In previous studies, the edge of the simulation domain was generally defined as a flux surface in the SOL and private region, and a straight line for the target region, as shown in Figure-1. This domain configuration is robust as it ensures Mach-1 Bohm boundary conditions (when the plasma flow is set to the sound speed) are only necessary on the target boundary.

The time stepping is done using the implicit Crank-Nicolson scheme, so that the size of time steps depends only on the time scale of the instabilities that are simulated. This implicit scheme 


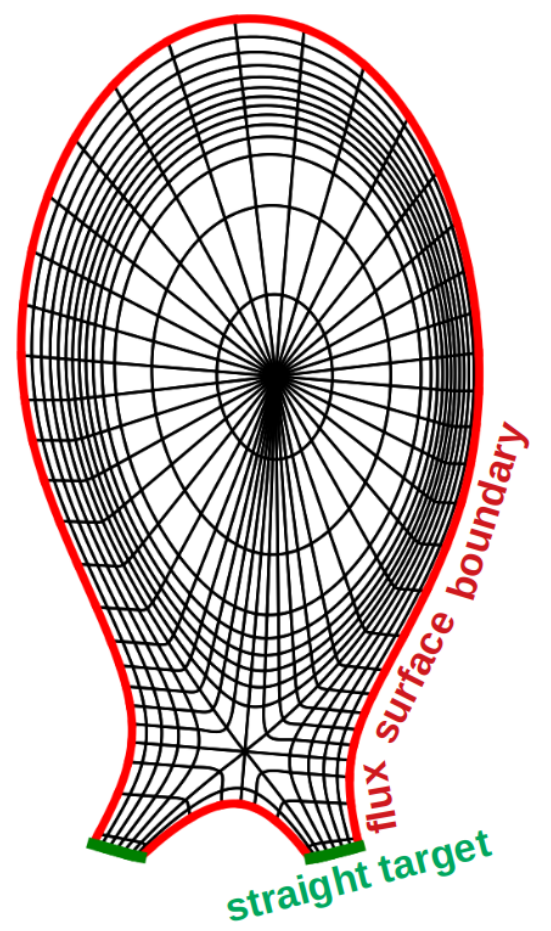

Figure 1: A typical lowresolution grid with $X$-point in the poloidal plane (the toroidal direction is normal to the paper). The domain boundaries are defined by flux surfaces in the SOL and the private regions (red), and by straight lines for the divertor targets (green). The confined region of the plasma is where flux surfaces are closed (inside the $X$ point contour), and the $S O L$ region is where flux surfaces are open onto the divertor target. More details on tokamak magnetic topology can be found in Figure-2 of [37]

results in a sparse system of equations, which is solved using a Generalized Minimal REsidual Solver (GMRES). The preconditioner for this iterative GMRES is obtained by solving independently each sub-matrix corresponding to different Fourier harmonics, which amounts to a block-Jacobi preconditioner. These sub-matrices are solved using the direct parallel sparse matrix solver PaStiX [38].

In order to allow the $n=0$ component of the $\vec{E} \times \vec{B}$ and parallel flows to evolve towards a stationary equilibrium, the simulations are first run without toroidal modes, with only the equilibrium $n=0$. This allows the kineteic equilibrium to reach a steady state consistent with the Bohm boundary on the target [9]. Note that at the zeroth time step, $\overrightarrow{\mathrm{v}}_{\|}$is Mach-1 on the target [9], and zero inside the plasma, already at the nodes adjacent to the boundary.

\section{The Wall-Extension Grid Generator}

In view of adressing immediate tokamak physics issues of various domains, ranging from divertor physics [33] to plasma-wall interactions during disruptions [39], the grid generator of JOREK has been generalised to allow the extension of a flux-aligned grid to any surrounding wall structures. The method developed for this purpose is described here to demonstrate its robustness.

The JOREK code builds a grid aligned to the $\psi$-map calculated from the Grad-Shafranov equilibrium, which is the result of balancing the plasma pressure $\nabla p$ against the magnetic forces $\vec{J} \times \vec{B}$ in the static stationary momentum equation:

$$
R^{2} \nabla \cdot\left(\frac{1}{R^{2}} \nabla \psi\right)=-\mu_{o} R^{2} \frac{d p}{d \psi}-F \frac{d F}{d \psi} .
$$




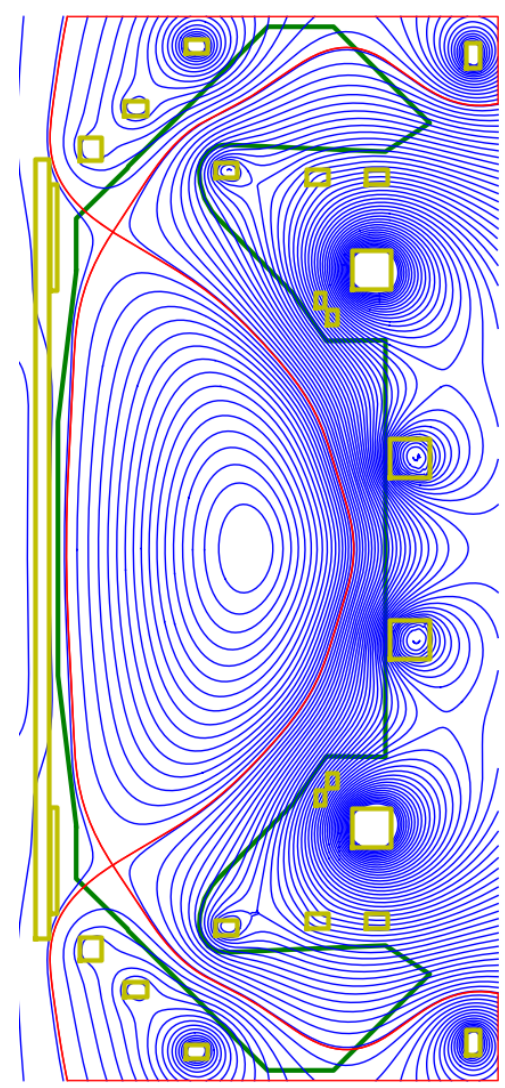

(a)

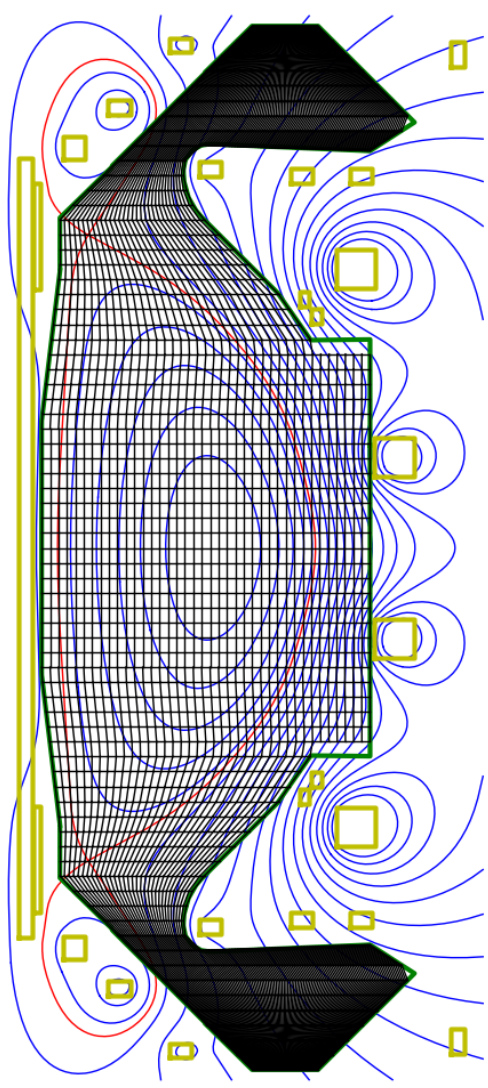

(b)

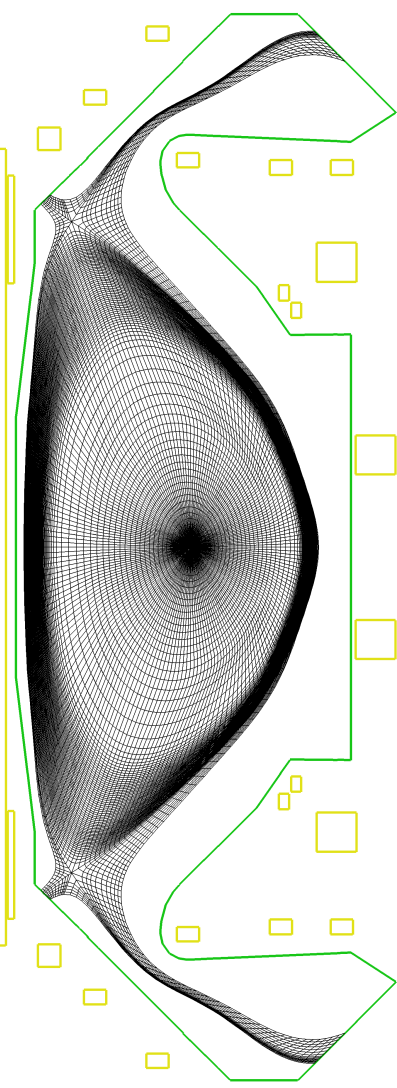

(c)

Figure 2:

(a) Magnetic flux contours (blue) for a Super-X configuration of the MAST-U device. The connected double-null separatrix contour is shown in red, the first-wall in green, and the PF-coils in yellow.

(b) The initial rectangular grid on which the JOREK Grad-Shafranov equilibrium is solved, to obtain a flux-map consistent with ballooning unstable pre-ELM pressure profiles. For this Grad-Shafranov equilibrium, the boundary condition is the flux taken from the input equilibrium (a). This initial rectangular grid is defined inside the first-wall, to avoid the necessity of taking PF-coil currents into account for the Grad-Shafranov equilibrium.

(c) The initial flux-aligned grid contained inside the first wall. This is the grid which can be extended. Note that a low-resolution grid is shown here for visual purpose.

JOREK can take any input for the initial Grad-Shafranov equilibrium, and use the exact pressure profile, the $F F^{\prime}$ profile (with $F^{\prime}=d F / d \psi$ ) and the $\psi$-map given from any equilibrium such as EFIT $++[40]$. However, in some cases, particularly ELM studies, it is desirable to use different pressure and $F F^{\prime}$ profiles than the ones provided by external equilibrium solvers, to ensure that the initial equilibrium is unstable with respect to peeling-ballooning modes. In these cases, the $\psi$-map from the original equilibrium is often inconsistent with the new pressure profile, which results in a significant imbalance between the $\nabla p$ term and the $\vec{J} \times \vec{B}$ term of equation (3). This would result in an inherently unstable equilibrium. To ensure this balance is respected, JOREK solves its own Grad-Shafranov equilibrium internally.

In previous cases, as for Figure-1, a polar grid was used to solve the new Grad-Shafranov equilibrium, for which the pressure and $F F^{\prime}$ profiles are given as input by the user, and the boundary condition for the solver is taken as the $\psi$-values of the original equilibrium data along 
the contour of this polar grid. The final flux-aligned grid is then built using the new equilibrium calculated on this polar grid. In the present case, since we aim at building a grid that extends to the wall, instead of a polar grid, a rectangular grid is built inside the first-wall, as shown in Figure-2b. This is convenient as it allows the fixed-boundary Grad-Shafranov solver to ignore all PF-coil currents outside the wall, so that the $\psi$-values along the wall are used as conditions for the fixed-boundary problem. Note that the equilibrium in Figure-2a was obtained using the FIESTA code [41], such that the $\psi$-values on the wall are consistent with the MAST-U coil-set.

Before extending a grid to the surrounding wall, we must build an initial grid inside that wall. This is done in a similar manner as in Figure-1, where the target must be aligned to the wall. An example of such initial grids is shown in Figure-2c, for a MAST-U Super-X configuration. Since the JOREK grid is composed of quadrilateral elements, an extension of a given grid must be composed of a number of quadrilaterals. Thus, the wall-extended grid is built simply by adjoining additional quadrilateral grid patches to the initial grid.

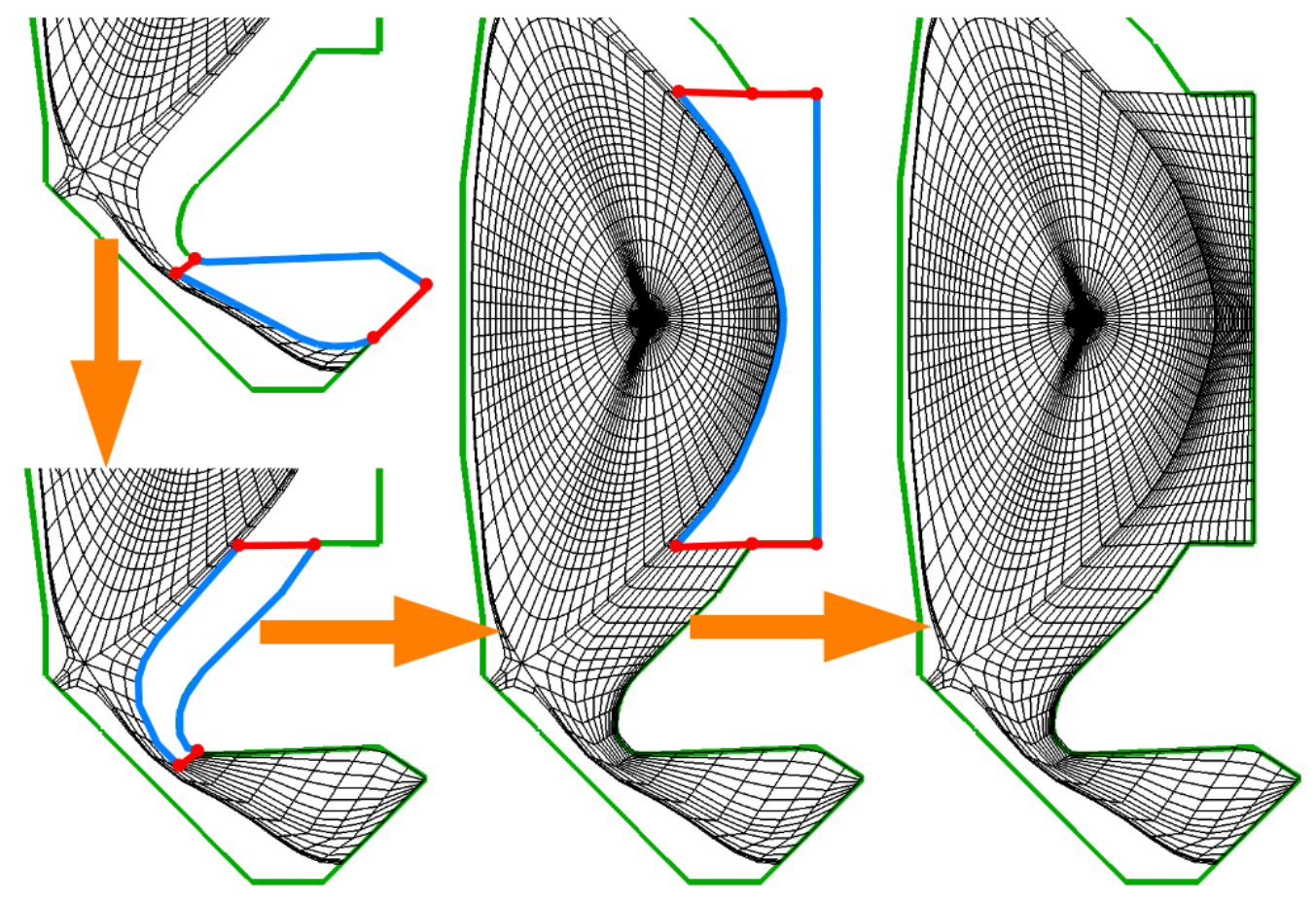

Figure 3: An example of successive grid patches applied between the flux-aligned X-point grid and the wall. Grid extension patches need to be quasi-quadrangles, but do not need to have linear sides. The user specifies the sides of the patch (red lines) using a set of input points (red points). The grid generator then automatically extends the grid between the grid side and the wall side (blue lines).

The flexibility of Bezier elements allows for grid patches to have non-linear sides, including curves and angles (although of course angles are best described if they lie on the vertex of an element). The idea of the new generator is that the user can provide near-quadrilateral patches between the initial grid and the wall. This is done by specifying the two sides of each patch, 
between the grid and the wall, like the red lines in Figure-3. Note that these side can be composed of multiple lines. The other two sides of the patch are determined automatically, along the grid, and along the wall, like the blue lines in Figure-3. The only requirement is that successive patches need to have matching sides. To ensure a smooth transition between the initial grid and the patches, the radial distribution of elements for the patches is automatically adapted such that their radial resolution at the junction matches that of the initial grid. Of course, the user can also specify the radial resolution of the new patches, but the poloidal resolution, however, is determined by the initial grid. In the case of matching successive patches, as in Figure-3, the radial resolution of the first patch determines the radial resolution of the following patches. Note that this patching method can be used any number of times, such that new grid patches can also be added on top of previous grid patches, which enables the description of complex wall structures, such as corners or isolated vacuum regions. The local refinement of Bezier elements, as in [26], could also allow improved numerical stability at geometrically complex boundary limits, but this particular feature has not been tested for the purpose of this study, and is left for future works.

In Appendix-A, further details are provided for each step of the grid construction and extension, including the method used to build the first grid with legs that extend up to the wall, the algorithm used to choose the nodes inside each patch, how to obtain a smooth transition of resolution between the previous grid and a new patch, and the simple method used to join grid patches together. Alternatively, a working copy of the grid generator is available from the JOREK code, upon request on https://www.jorek.eu. However, since this grid generator is tightly linked to the Bezier FEM formulation used in JOREK, it cannot simply be extracted as such, and further work would be required to make it generic and applicable to any code. In particular, the grid-generator requires an accurate calculation of $\psi$-contours, which is simplified here due to the bi-cubic representation of $\psi$ within each element. The extension of this work to arbitrary grid representation would mostly involve the restructuration of the $\psi$-contour solver, since all other methods used are largely independent of the FEM formulation.

This grid-extension method is generic and robust enough that it may be applied to any toroidally axisymmetric tokamak device. Figure- 4 shows examples of extended grids for MAST-U, JET-ILW and JT-60SA, which all have very different wall contours and separatrix shapes.

In Section-5, the robustness of the grid is demonstrated and thoroughly tested using nonlinear simulations of highly unstable peeling-ballooning modes. Additionally, the JOREK code also solves the nonlinear elliptic partial differential equation for the Grad-Shafranov equilibrium, in order to calculate the precise values of each variable required for the physics model (equation-3-8). The convergence of the JOREK Grad-Shafranov solver, using the fixed-point iteration method, provides a preliminary demonstration of the robustness of thenew grid-generator. For example, using the JET plasma in Figure-4b (also used in Section-5), the equilibrium solver converges to an averaged accuracy of $10^{-7}$ with: 16 iterations using the original grid topology as in Figure1 (no wall-extension), and 19 iterations using the wall-aligned grid shown in Figure-4b. While this remains reasonably fast, in non-linear simulations, convergence and numerical stability for 


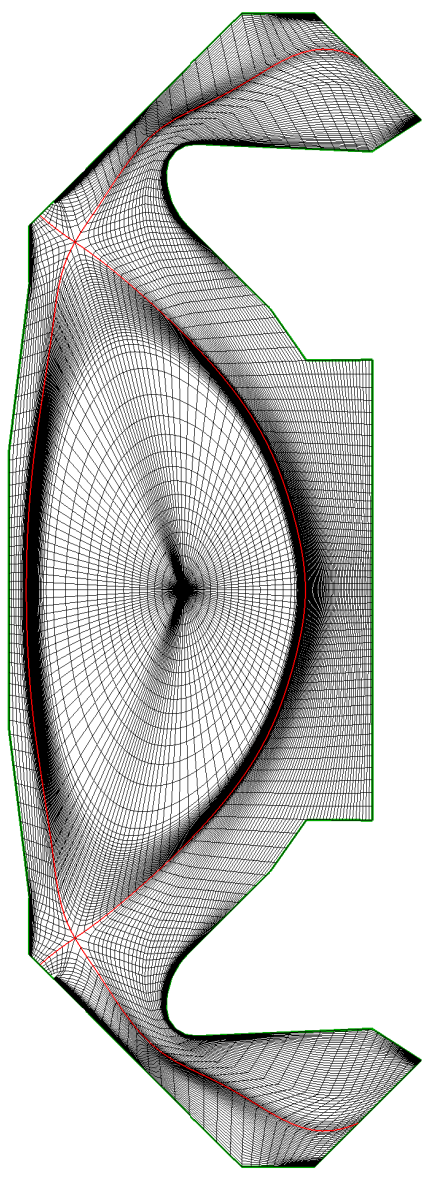

(a)

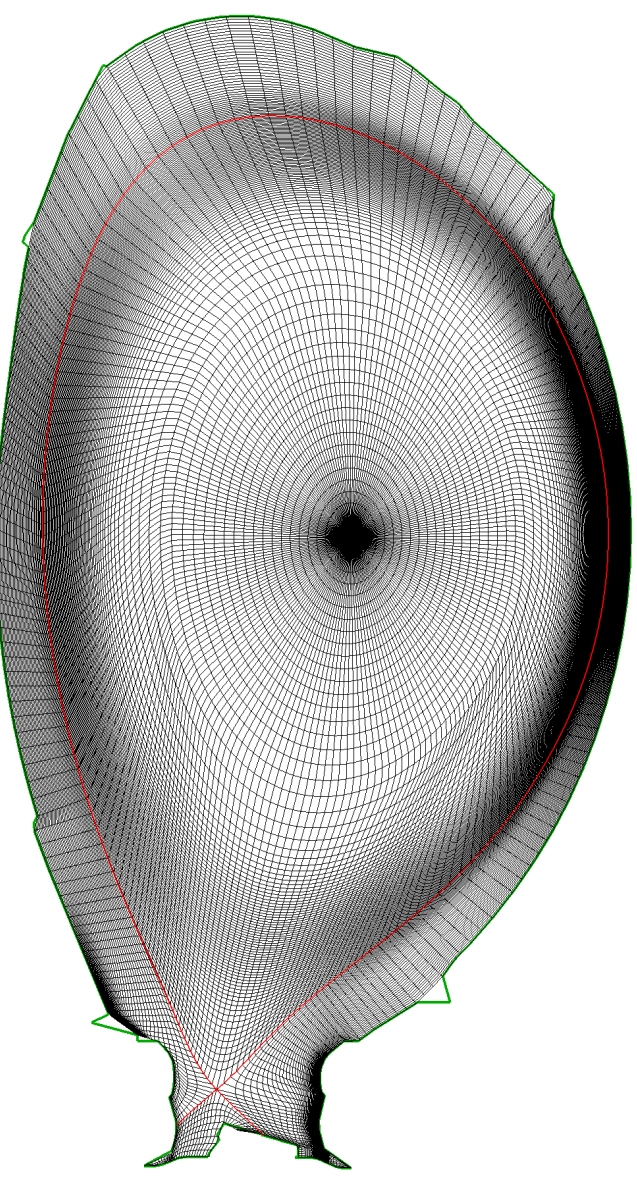

(b)

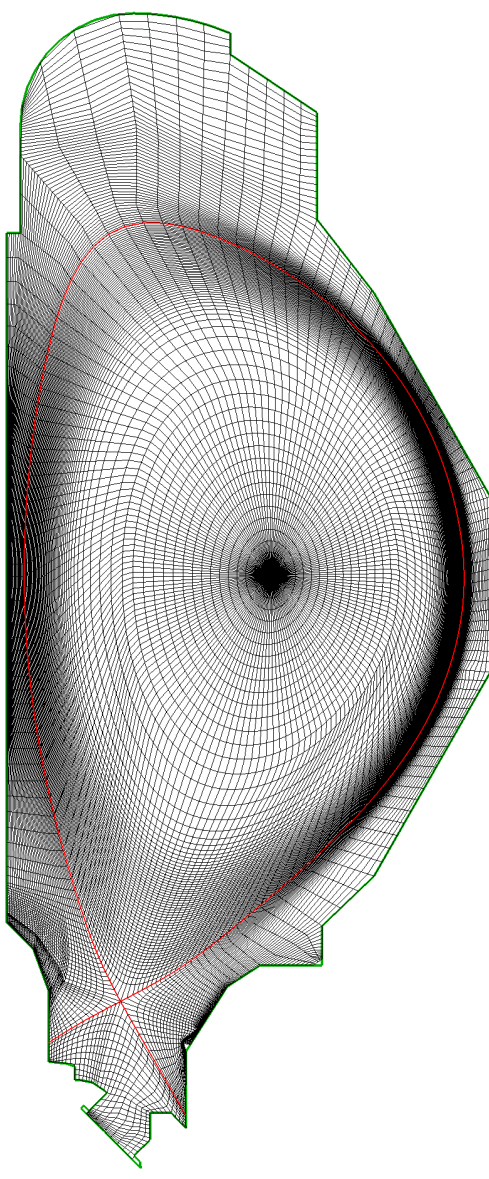

(c)

Figure 4: Examples of wall-extended grids for three devices: (a) MAST-U, (b) JET-ILW and (c) JT-60SA.

wall-aligned grids strongly depends on the robustness of boundary conditions, which cannot be evaluated by the Grad-Shafranov solver.

\section{Sheath Boundary Conditions with a Wall-aligned Grid}

With this new wall-extended grid, appropriate boundary conditions are required. The usual case is that magnetic field lines are incident to the boundary surfaces, thus requiring Bohm (Mach-1) and Sheath boundary conditions [9]. These are expressed as:

$$
\begin{aligned}
& \overrightarrow{\mathrm{v}}_{t o t} \cdot \vec{n}= \pm c_{s}=\sqrt{\gamma T} \vec{b} \cdot \vec{n} \\
& n T \overrightarrow{\mathrm{v}}_{\|}+\kappa_{\|} \nabla_{\|} T=\gamma_{s h} n T \overrightarrow{\mathrm{v}}_{\|} .
\end{aligned}
$$

where $\vec{n}$ is the unit vector normal to the boundary, $\vec{b}$ is the unit vector along the magnetic field, $\gamma=5 / 3$ and $\gamma_{s h}=4.5$. In the standard model, without neutrals density, density and temperature have free outflow boundary conditions at the target (no density reflection or recycling). However, when using the neutrals density model, as described above, a reflective (or recycling) coefficient $\xi_{\text {re }}$ 
can be applied at the boundary, such that density arriving at the target is (fully or partly) reflected as neutrals into the simulation domain, as in [42]:

$$
D_{n} \nabla \rho_{n} \cdot \vec{n}=-\xi_{r e} \rho \overrightarrow{\mathrm{v}}_{\|} \cdot \vec{n}
$$
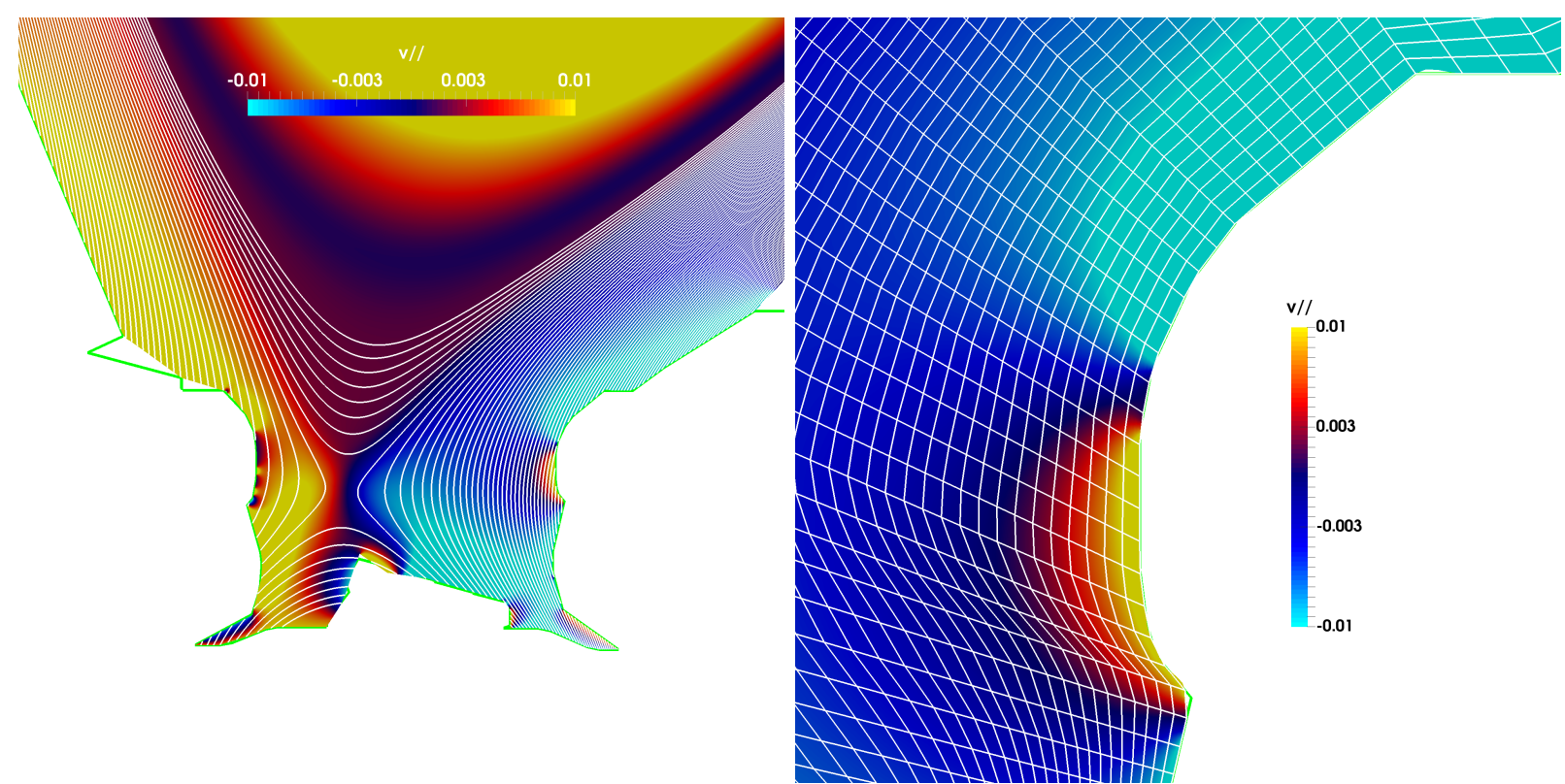

(a)

(b)

Figure 5:

(a) The direction of Mach-1 boundary conditions is determined by the direction of field lines going in/out of the domain boundary, which can change along the wall,

(b) The change in direction of the Mach-1 boundary condition can occur within a few elements.

Note that the amplitude of the velocity is in normalised units, such that 0.01 corresponds to approximately $20 \mathrm{~km} / \mathrm{s}$.

It should be noted that the direction of the magnetic field may change as we step along the boundary of the domain, such that the sign of $\vec{b} \cdot \vec{n}$ may be going from positive, across zero, to negative. This is illustrated in Figure-5, where the velocity is in normalised units, such that 0.01 corresponds to approximately $20 \mathrm{~km} / \mathrm{s}$. It implies that the Mach-1 boundary condition can reverse direction within a grid element. A threshold is used for the application of Mach-1 boundary conditions, which requires that the angle $\alpha$ between the magnetic field and the boundary is larger than $3^{\circ}$, which is approximately twice the lower limit given by Geraldini et al. [43]:

$$
\sqrt{\frac{m_{e}}{m_{i}}} \ll \alpha \ll 1
$$

If the angle $\alpha$ is smaller than $3^{\circ}$, then instead Dirichlet boundary conditions are applied to $\psi$, $\Phi, \overrightarrow{\mathrm{v}}_{\|}$and $j$, while Neumann boundary conditions are applied to $\rho, T$ and $\rho_{n}$. Even with relatively low parallel viscosity for equation-4, simulations remain numerically stable. In the ELM simulation presented in the next section, a spacially uniform value of $\mu_{\|}=10^{-6} \mathrm{~kg} \cdot \mathrm{m}^{-1} \cdot \mathrm{s}^{-1}$ was used. A relatively small value of $\mu_{\|}$was used here to test the numerical robustness of the simulations, since 
larger $\mu_{\|}$values should in principle be numerically easier. In the SOL, at about $10 \mathrm{eV}$, the Braginskii viscosity is approximately $1.5 \times 10^{-5} \mathrm{~kg} \cdot \mathrm{m}^{-1} \cdot \mathrm{s}^{-1}$. Note that more advanced boundary conditions, such as in [44], would be desirable for such wall-aligned grids, particularly for diamagnetic drifts, but this is left for future developments.

\section{Current Physics Applications with the Wall-extended Grids}

\section{First-wall and divertor heat-fluxes}

In order to demonstrate the ability of the new wall-extension grid-generator to handle non-linear simulations of large type-I ELM crashes, a type-I ELMy H-mode experiment of JET-ILW has been used. Pulse JPN-83334, which was thoroughly simulated and analysed in [27], is a 2.4T, 2.4MA, low-triangularity plasma, with 25MW of NBI heating, and with the outer strike point positioned on the main (bulk) tungsten divertor tile. The pre-ELM pedestal electron density and temperature are $5 \cdot 10^{19} \mathrm{~m}^{-3}$ and $1.2 \mathrm{keV}$ respectively, which is unstable with respect to ideal MHD ballooning modes. For this test simulation, relatively high MHD parameters were used: a resistivity 200 times higher than $\eta_{\text {spitzer }}$, and a viscosity (both parallel and perpendicular) of $10^{-6} \mathrm{~kg} \cdot \mathrm{m}^{-1} \cdot \mathrm{s}^{-1}$, and diamagnetic effects are not included. The toroidal resolution used was $n=2,4,6,8,10$. More challenging simulations with the wall-extended domain, using higher toroidal resolutions and more challenging diffusive parameters, will be the focus of future research in the coming years.

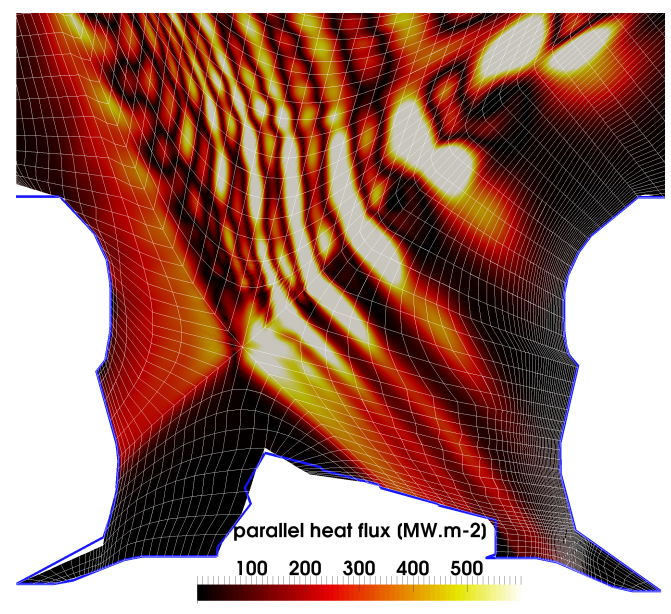

(a)

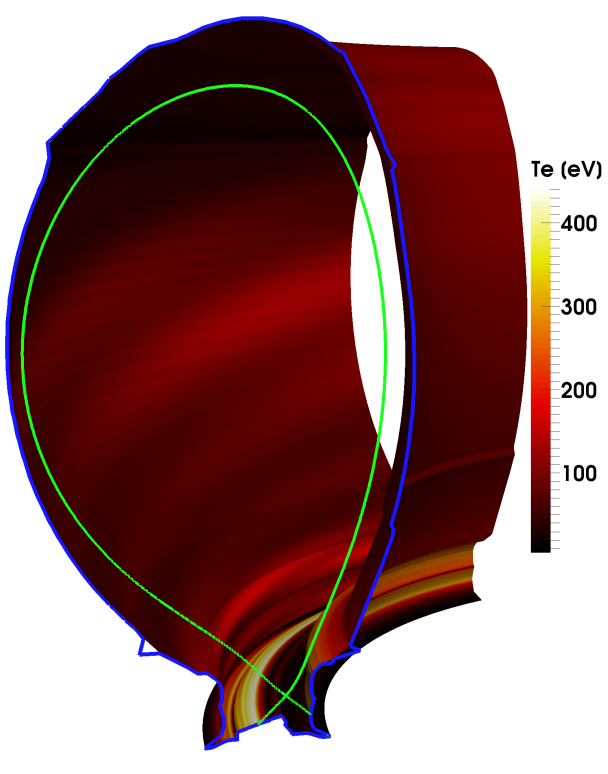

(b)

Figure 6:

(a) The parallel heat-flux amplitude in a JET-ILW ELM simulation,

(b) The electron temperature $T_{e}[\mathrm{eV}]$ of the plasma at the wall boundary during an ELM simulation in JET-ILW.

The energy loss of this simulated ELM is $3.9 \%$ of the total plasma energy content, which 
is reasonably close to the experimental value of $4.6 \%$ (averaged of all ELMs in the discharge). The peak heat-flux on the divertor target reaches just above $380 \mathrm{MW} \cdot \mathrm{m}^{-2}$, which is also close to the experimental value of $360 \mathrm{MW} \cdot \mathrm{m}^{-2}$ (also averaged over all ELMs), and close to values found previously in [27]. Note that, while reasonable agreement is achieved between this simulation and the corresponding experiment, the purpose of this work is not to validate the JOREK simulations (which would require diamagnetic effects as well as lower resistivity and viscosity), but rather to demonstrate the robustness of the new wall-extended grid and its applicability to simulations of type-I ELM instabilities. Figure-6a shows the parallel heat-flux for a snapshot of this simulation, while Figure-6b shows the temperature of the plasma at the first wall boundary at this same snapshot. Detailed comparisons between wall-grids and non-extended grids are now under way, but will be the subject of future publications. In particular, whether the $\gamma_{s h}$ parameter of equation-12 has any influence on the energy losses during an ELM remains an open question.

\section{Divertor configurations, gas-fueling, and detachment}

One of the main interests of providing an accurate description of the first-wall boundary in JOREK simulations is to enable the simulation of various gas-puffing levels as well as divertor configurations. As described in [45], JET-ILW experiments have demonstrated the importance of gas-puffing levels regarding ELM dynamics as well as global confinement levels. Similarly, the separatrix position with respect to the gas-injection valves and the divertor pumps plays a major role in neutrals dynamics and, as a consequence, pedestal performance in JET-ILW experiments [46].

Although the neutrals model described in section-2.1 and in [28] may not be sufficient to fully describe the complete neutrals dynamics in the SOL of JET-ILW (or other devices), it could provide a preliminary insight into the effect of SOL neutrals levels on pedestal and ELM physics. Figure-7 shows a test simulation of an ELM using the full model described in Section-2.1. The interaction of plasma filaments with the neutrals background is clearly visible in the upper SOL region. Note that this is a demonstrative simulation, and that the neutrals background of the SOL may be higher in the experiments, which would affect how ELM filaments interact with the neutrals in the SOL. Such detailed physics studies could be addressed in future works.

Further work is under way to benchmark JOREK simulations of neutrals simulations with SOLPS [47], particularly addressing simulations of detachment and ELM burn-through in the new MAST-U device [48, 49]. As shown in Figure-4a, the detailed description of the entire plasma domain enables the representation of increased neutrals pressure inside the closed divertor leg, crucial for detachment studies, as well as the computation of heat-fluxes on the nose of the divertor (where the outer wall almost reaches the outer leg, just below/above the X-points). However, this is a continuing effort which is left for future publications. In addition, a project is underway to add kinetic neutrals as particles [50]. 

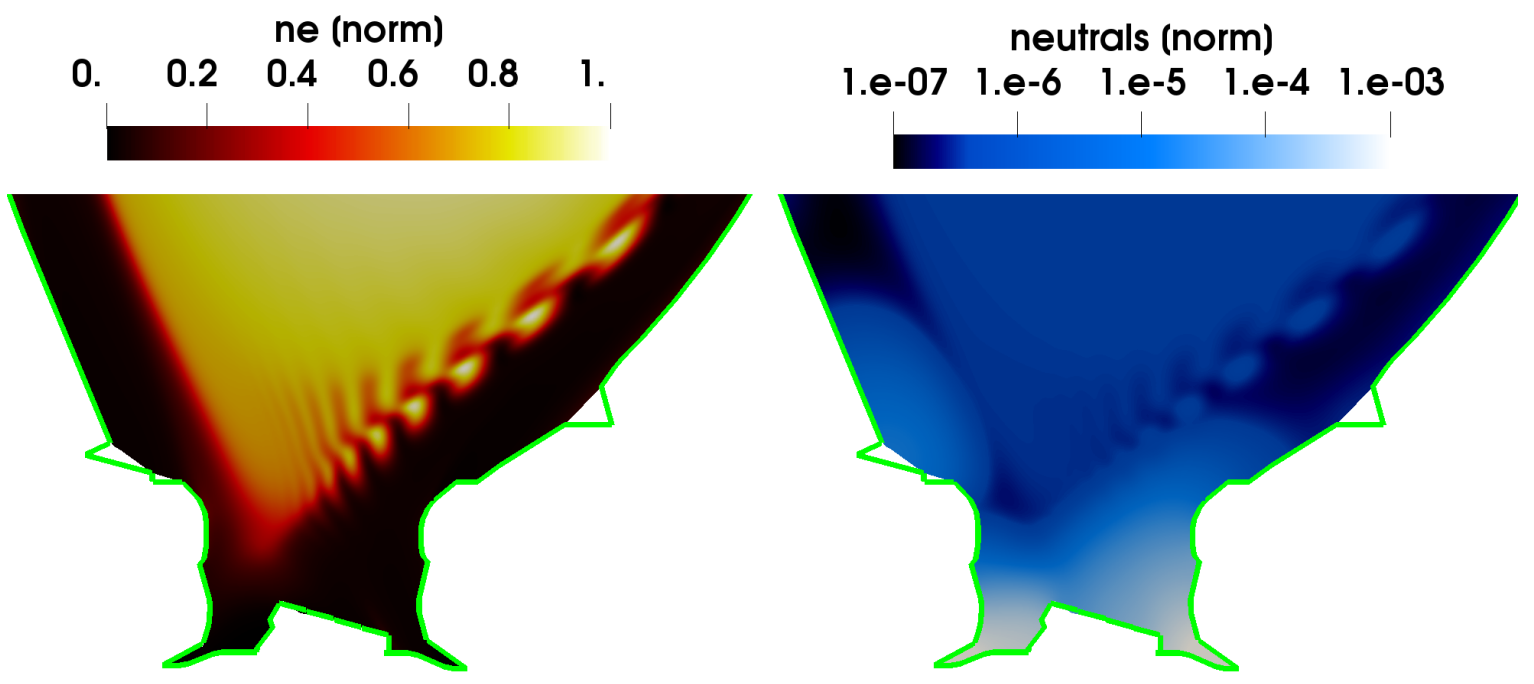

Figure 7: A simulation of ELM filaments using the neutrals density model with divertor reflection of neutrals. Electron density (left) and neutrals density (right) are shown in the divertor region. Both quantities are normalised to the central $n_{e}$ value: $0.65 \times 10^{20} \mathrm{~m}^{-3}$.

\section{Summary and Future Studies}

A new grid generator has been developed for the JOREK code, enabling any equilibrium to be meshed, including the entire plasma domain, all the way to the first wall. The appropriate fluid boundary conditions are applied to all wall surfaces, providing a detailed description of SOL flows. This paper demonstrates the practical feasibility of building grids for various tokamak devices, and an advanced JET-ILW ELM simulation shows the robustness of such new grids when used for state-of-the-art non-linear MHD simulations.

Block-structured mesh generation is widely used in the CFD community, and this work is part of a common effort to bring this technique to the fusion community [24, 25]. The method developed here is adapted to the quadrilateral topology imposed by rectangular Bezier elements, and so it may be of interest to the wider scientific community, beyond tokamak physics.

The wide range of physics areas accessible with this new grid generator is duplicated by its coupling with the JOREK neutrals density model. Advanced studies of divertor, SOL and pedestal physics, including detachment, ELM burn-through and impurity transport, can now be done in a detailed and systematic manner. This work also contributes a significant step towards the full 3D description of plasma-wall interactions in disruption studies with the JOREK-STARWALL code $[39,51]$. The goal of detailed 3D tiled-wall representation, to provide precise calculations of hiro and halo currents during disruptions, is a long-term challenge, and one of ITER's and DEMO's most pressing issues. Figure-8, which shows a JOREK simulation with ELM filaments (left), compared to the visible camera during a JET experiment (right), gives a good notion of the level of three-dimensional details that would be required for an accurate description of the actual plasma facing components. How such detailed geometry affects the plasma behaviour remains a largely unexplored issue of tokamak physics. 


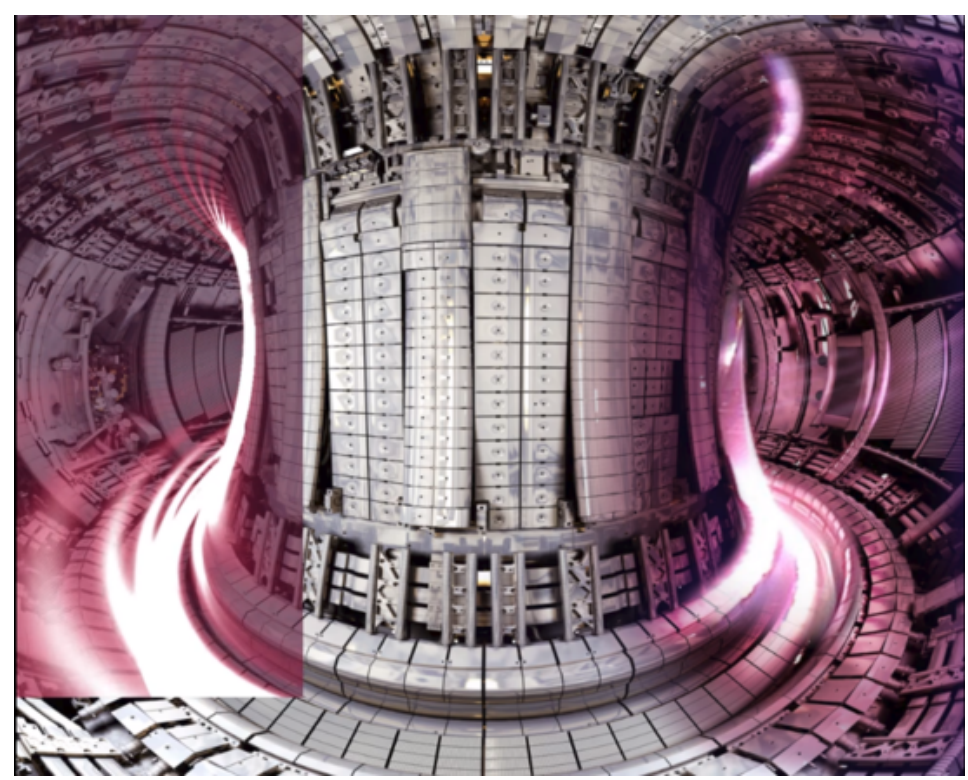

Figure 8: A synthetic diagnostic of JET fast-visible camera for a JOREK ELM simulation (left), compared to an experiment (right). This picture represents well the surprisingly high level of 3-dimensional details required for an accurate description of the wall. This work is a first step towards such detailed spatial discretizations.

\section{Acknowledgement}

This work has been carried out within the framework of the EUROfusion Consortium and has received funding from the Euratom research and training programme 2014-2018 under grant agreement No 633053, and from the RCUK Energy Programme [grant number EP/I501045]. To obtain further information on the data and models underlying this paper please contact PublicationsManager@ccfe.ac.uk. This work used the HELIOS supercomputer (IFERC-CSC), Japan, under the Broader Approach collaboration, implemented by Fusion for Energy and JAEA. The views and opinions expressed herein do not necessarily reflect those of the European Commission or the ITER Organization. The HEC ARCHER computer (UK), as part of the Plasma HEC Consortium EPSRC grant EP/L000237/1, and the MARCONI computer at CINECA in Italy, were also used. Finally, the PRACE project Tier-0 JVSITPEI has provided support on the MareNostrum HPC-cluster at BSC-Barcelona, Spain.

\section{References}

[1] A.S. Kukushkin et al. A. Loarte, B. Lipschultz. Nucl. Fusion 47, S203-S263, 2007.

[2] J.W. Coenen et al. Journal of Nuclear Materials 463, 78-84, 2015. 
[3] J.A. Goetz et al. Phys. Plasmas 6, 1899, 1999.

[4] S.I. Braginskii. Review of Plasma Physics Vol.1, Ed M.A. Leontovitch (New York: Consult. Bureau) p205, 1965.

[5] R. Goldston. Phys. Plasmas 17, 012503, 2010.

[6] A. Scarabosio et al. T. Eich, B. Sieglin. Phys. Rev. Lett. 107, 215001, 2011.

[7] R.A. Pitts et al. Journal of Nuclear Materials 438, S48-S56, 2013.

[8] M. Merola. Journ. Nucl. Materials 2, 307-311, 1524-1532, 2002.

[9] P.C. Stangeby. The Plasma Boundary of Magnetic Fusion Devices, Institute of Physics Publishing, 2000.

[10] A. Kirk et al. Plasma Phys. Contr. Fusion 48, 2006.

[11] B. Dudson et al. Plasma Phys. Contr. Fusion 50, 2008.

[12] S.J. Zweben et al. Plasma Phys. Control. Fusion 49, S1-S23, 2007.

[13] S.J. Zweben et al. Nucl. Fusion 44, 134-153, 2004.

[14] B.D. Dudson and J.Leddy. Plasma Phys. Control. Fusion 59, 054010, 2017.

[15] R.Schneider et al. Contrib. Plasma Phys. 46, 3, 2006.

[16] P.Tamain et al. J. Comp. Phys. 321, 606-623, 2016.

[17] C.S.Chang et al. J. Phys.: Conference Series 180, 012057, 2009.

[18] A.Stegmeir et al. Plasma Phys. Control. Fusion 60, 035005, 2018.

[19] G.T.A.Huysmans and O.Czarny. Nucl. Fusion 47, 659, 2007.

[20] G.T.A.Huijsmans and A.Loarte. Nucl. Fusion 53, 123023, 2013.

[21] H.K.Versteeg et al. An introduction to computational fluid dynamics : the finite volume method (2nd ed.). Harlow: Prentice Hall. ISBN 9780131274983, 2007.

[22] W.L.Chen et al. Comp. Meth. in Appl. Mech. and Engin. 144, 3-4, 1997.

[23] B.Wang et al. Journal of Aerospace Comp. 5, 11, 2008.

[24] H.Guillard et al. Tokamesh : A software for mesh generation in Tokamaks. HAL Id: hal01948060. https://hal.inria.fr/hal-01948060, 2018.

[25] O.Izacard et al. Gingred, a general grid generator for $2 D$ edge plasma modeling. https://arxiv.org/pdf/1705.05717.pdf, 2018.

[26] O.Czarny and G.T.A.Huysmans. J. Computational Phys. 227, 7423, 2008.

[27] S.Pamela et al. Nucl. Fusion 57, 076006, 2017.

[28] A.Fil et al. Physics of Plasmas 22, 062509, 2015.

[29] F.Orain et al. Phys. Plasmas 20, 102510, 2013.

[30] H.R. Strauss. Phys. Fluids 19, 134, 1976.

[31] O.Sauter et al. Phys. Plasmas 6, 7, 1999.

[32] H.P.Summers. http://adas.ac.uk, Atomic Data and Analysis Structure User Manual.

[33] M. Blommaert et al. Contrib. Plasma Phys. 58, 718-724, 2018.

[34] E.Nardon et al. Plasma Phys. Control. Fusion 59, 014006, 2017.

[35] H.R. Strauss. J. Plasma Physics, Vol. 57, part 1, pp 83-87, 1997.

[36] E.Franck et al. ESAIM:M2AN 49, 5, https://doi.org/10.1051/m2an/2015014, 2015. 
[37] S.Pamela et al. Plasma Phys. Control. Fusion 55, 095001, 2013.

[38] P. Henon et al. Parallel Comp. 34 345-362, 2008.

[39] F.J.Artola et al. Nucl. Fusion 58, 096018, 2018.

[40] L.Appel et al. Computer Physics Communications 223, 1-17, 2018.

[41] L.Pangione et al. Fusion Eng. Des. 88, 1087, 2013.

[42] M.Verbeek et al. 43rd EPS Conference Proceedings, P5.058, 2016.

[43] A.Geraldini et al. Plasma Phys. Control. Fusion 59, 025015, 2017.

[44] J.Loizu et al. Phys. of Plasmas 19, 122307, 2012.

[45] C.Maggi et al. Nucl. Fusion 55, 113031, 2015.

[46] E.Joffrin et al. Nucl. Fusion 57, 086025, 2017.

[47] D.Moulton et al. Plasma Phys. Control. Fusion 59, 065011, 2017.

[48] S.Smith et al. 45th EPS conference, Prague, Czech Republic, 2018 http://ocs.ciemat.es/EPS2018PAP/pdf/P4.1061.pdf.

[49] S.Pamela et al. 27th IAEA-FEC conference, IAEA-CN-258/OV/4-4, 2018.

[50] D. Van Vugt et al. 45th EPS conference, Prague, Czech Republic, 2018 http://ocs.ciemat.es/EPS2018PAP/pdf/P1.1049.pdf.

[51] M.Hoelzl et al. Journal of Physics: Conference Series 401, 012010, 2012. 


\section{Appendix-A: Details of the grid-to-wall Extension Method}

This Appendix gives some details of the methods used for the key aspects of the grid-generator which extends a grid to the tokamak wall. The implementation of the generator can be available upon request by contacting JOREK experts on https://www.jorek.eu.

\section{Joining grid-patches together}

One of the central routines of the new grid generator is simply to join different grid patches together. This is straight-forward and uses the fact that element nodes are labelled if they lie on the boundary of the domain, which is determined simply by checking how many elements a node belongs to: unless it belongs to 4 elements, it is a boundary node. Looping around the boundary of the main grid patch, each node position is compared to each one of the second grid patch. If their position matches within $10^{-5} \mathrm{~m}$, the node is included into a list of 'common nodes'. The grid elements and nodes of the second patch are then appended to the first one, with the exception of the "common nodes'. Note that we do not check whether the two grid patches overlap, since this should not occur in the present context.

When joining a new patch to the main grid, the 'common nodes' will, by definition, need to be the same. In practice, this means that the resolution of the new patch is determined by the resolution along the boundary of the main grid. Thus, only the radial resolution can be chosen by the user. For cases where the new patch needs to be aligned on two of its sides, like the second patch of Figure-3, the resolution of the patch in both directions is determined by the main grid.

\section{Building the initial grid with wall-extended divertor legs}

Details of the construction of $\psi$-aligned grids are already provided in [37], however for this new grid generator, the original method was slightly modified to improve the robustness of the generator. The initial grid, without the wall-extending patches, is constructed in two parts: the central part (above and below the lower and upper X-point respectively), and the divertor legs. The construction of the central grid is similar to the one described in [37]. Once the central grid is built, each divertor leg is constructed independently, by segmenting $\psi$-contours between the central grid and the wall. Both lower legs are then patched together, and then patched to the main grid (similarly for the upper divertor legs). This is illustrated in Figure-9.

The divertor legs are extended up to the divertor by segmenting the length of $\psi$-contours between the central grid limit and the wall, and grid elements are thus determined between the segments of two $\psi$-contours. This segmentation allows for a compression of the resolution near the X-point and/or near the wall, which is important for accurate description of Mach-1 and Sheath boundary conditions (see Section-4). 


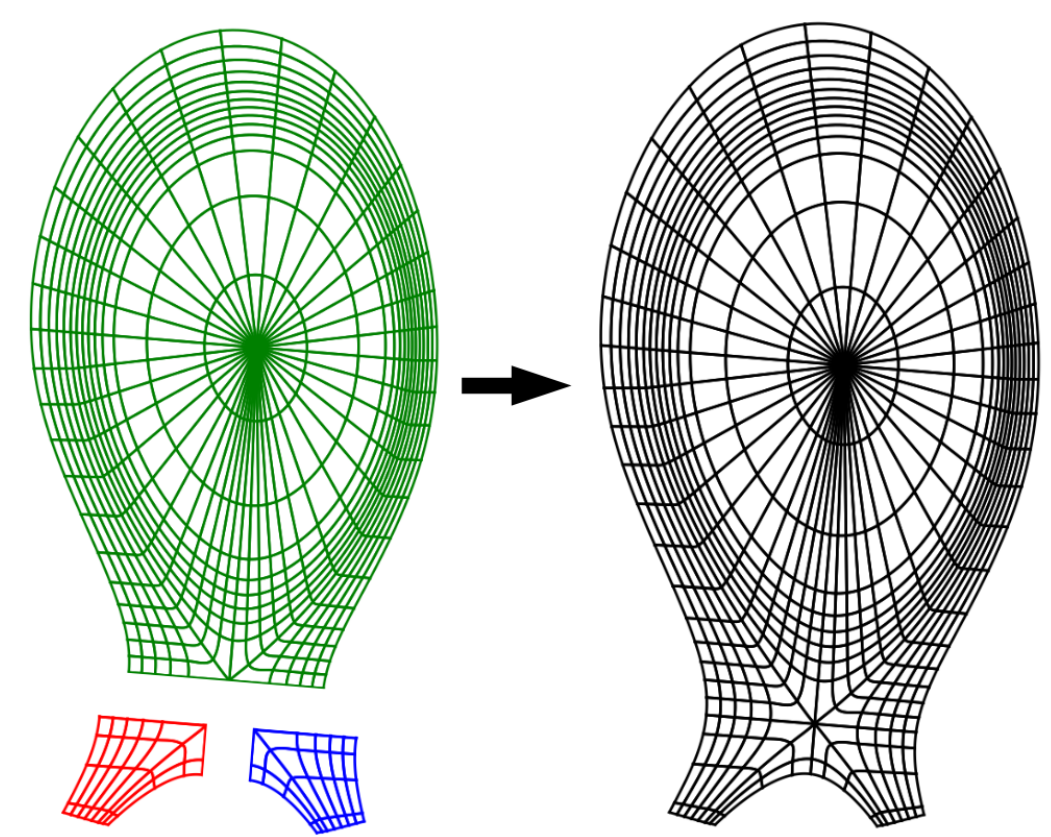

Figure 9: The initial X-point grid, before the wall extension, is built in two parts: the main plasma region (green) and the divertor legs (red and blue). These are then patched together to form the X-point grid (black). Building each leg individually allows more flexibility to extend them all the way to the wall of the tokamak.

\section{Obtaining a smooth transition between the main grid and a new patch}

The transverse resolution of a new patch can be compressed or expand at the border with the main grid. Typically, we assume that $1 / 3$ of the transverse width of the patch is sufficient to allow for this compression/expansion to provide a smooth transition. However, depending on the requested transverse resolution, this can lead to a compression/expansion of resolution at the other end of the patch (ie. on the wall), particularly if the transverse width of the patch varies strongly along its length. Such an example can be seen in Figure-4a and is reproduced with a zoom in Figure-10a, showing both cases: a compression of resolution on the wall (green arrow), and an expansion of resolution on the wall (red arrow). Generally, a higher resolution on the wall is preferable, such that the latter case should be avoided. One additional improvement for future developments would be to add user parameters, for each patch, to control the compression parameters of the radial resolution.

\section{Defining elements inside an arbitrary patch}

To obtain smooth transitions of resolutions between grid patches, the grid patches themselves must allow for a variation of resolution between the 4 edges of the patch. The method used to obtain this can be explained as follows. Firstly we assume that the resolution in both directions of the patch is known, since it is determined by the user and/or the main grid onto which we are attaching the patch, as explained above. Secondly, we parametrise the interior of the patch based on its edges (which are known). Thirdly, we segment the parametrisation variables using the requested resolution, and use the resulting values to obtain the positions of nodes on the final patch. 
Let the 2D patch be parametrised by $(s, t)$ such that any point of the patch is determined by $\mathbf{P}(s, t)=\left(P_{x}(s, t), P_{y}(s, t)\right)$ with $0.0 \leq s \leq 1.0$ and $0.0 \leq t \leq 1.0$. Since the edges of the patch are known, they may be used to define the parametrisation $\mathbf{P}(s, t)$ as:

$$
\mathbf{P}(s, t)=(1-t) \mathbf{P}(0, t)+t \mathbf{P}(1, t)+(1-s)[\mathbf{P}(s, 0)-\mathbf{L}(s, 0)]+s[\mathbf{P}(s, 1)-\mathbf{L}(s, 1)]
$$

where $\mathbf{L}(s, 0)$ is the straight line segment between $\mathbf{P}(0,0)$ and $\mathbf{P}(1,0)$, and likewise $\mathbf{L}(s, 1)$ the straight line segment between $\mathbf{P}(0,1)$ and $\mathbf{P}(1,1)$. This is illustrated in Figure-10b.

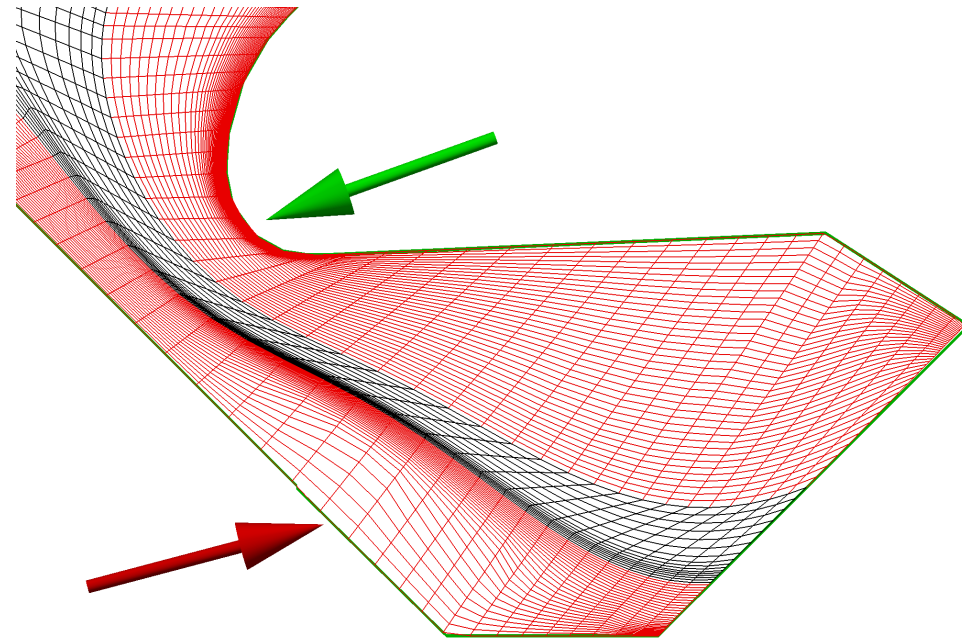

(a)

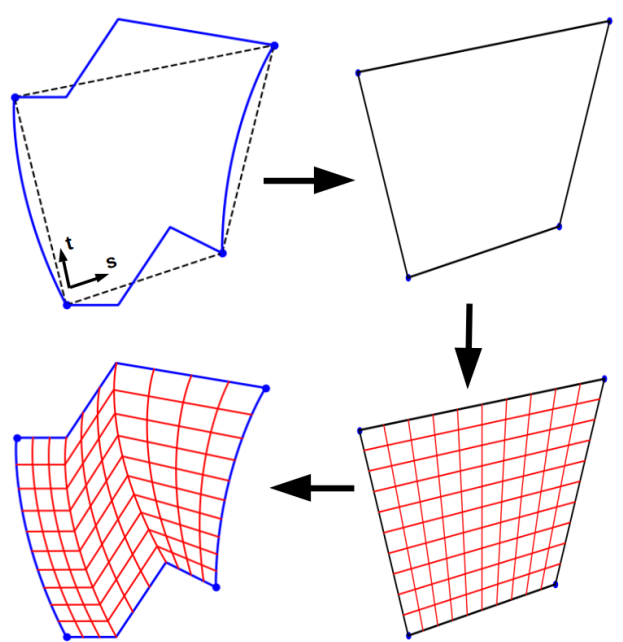

(b)

Figure 10:

(a) In order to obtain a smooth transition of resolution between the grid and new patches, it is typically necessary to compress or expand the resolution near the other end of the patch (ie. at the wall). The two cases are represented here. The resolution of new patches (red) needs to match the main $\psi$-aligned grid (black). This results in a compression of resolution near the wall on one side (green arrow), and in an expansion of the resolution on the other side (red arrow),

(b) Since the edges of the patch are know, they can be used to define a parametrisation and segment the patch into the requested mesh in a smooth manner. 